surface fault in the area of the aftershocks $2,6,7,8$ and 11 in Fig. 1, which is the area of the ground deformation in the Salayani-Alam valley. There were a few events south of the line Ferdos to Dashti Biaz, and a few scattered all over the area, some at a considerable distance from the fault.

A preliminary survey of events towards the end of the 4 weeks of recording suggests that they follow the same basic pattern. This pattern, broadly speaking, is what is to be expected as the crust readjusts after a shallow earthquake. The restricted area of the events and their close association with the surface faulting also point to the original earthquake being shallow.

STUART CRAMPIN GeORge ANDERson

Institute of Geological Sciences, MoHammad SHaHIDI

Geophysical Laboratories,

Edinburgh.

Received Xovember 1, 1968.

\section{A Triggered Spark Gap in Liquid Dielectrics}

WE are investigating the behaviour of a trigatron spark gap in liquid dielectrics. Because we believe that a triggered spark gap in liquids has not been investigated before, a few characteristics of this trigatron are described here.

The trigatron consisted of a spherical and a hemispherical electrode, both of $\frac{3}{4}$ inch diameter and made of stainless steel. A 0.035 inch diameter stainless steel rod mounted flush with the main electrode formed the trigger electrode, the trigger discharge gap being approximately 0.005 inch. The trigatron was mounted with its axis vertical in the test cell, which was filled with hexane of commercial grade.

If the d.c. breakdown voltage of the trigatron spark gap in the absence of a trigger pulse is $V_{\mathrm{BD}}$, the trigatron gap will break down over a range of voltages from $V_{\mathrm{BD}}$ down to some lower limiting voltage $V_{\min }$, when the trigger pulse $v$ is applied to the trigger electrode. According to the polarities of the voltage on the main gap, $V$, and the trigger pulse voltage, $v$, there will be four possible test combinations. Characteristics showing $V_{\min }$ as a function of gap spacing for all polarity combinations are shown in Fig. 1. They were obtained with trigger pulses of $v=5 \mathrm{kV}$ produced by an impulse generator having a $0.04 \mu \mathrm{F}$ capacitor. These tests were made with the circuit arrangement shown in Fig. 2.

The breakdown voltage of the trigatron spark gap seemed subject to statistical fluctuations. The results were obtained by noting the number of breakdowns for a series of 50 trigger pulses at each voltage $V$ applied on the main gap, and $V_{\text {min }}$ was taken as the voltage for which 50 per cent of the trigger pulses caused breakdown. Fig. 1 also shows the breakdown voltage $V_{\mathrm{BD}}$ of the trigatron spark gap for both polarities as a function of gap setting.

It was found that triggered breakdowns occurred at main gap voltages which were less than half the normal untriggered breakdown voltage. The reduction in breakdown voltage is greater when the main gap polarity is negative (that is, the spherical electrode is negative), just as Sletten and Lewis ${ }^{1}$ found for trigatrons in air. $V_{\min }$ for the liquid trigatron, however, is dependent on the polarity of $v$ for both polarities of $V$, whereas for the air trigatron $V_{\min }$ is only dependent on the polarity of $v$ when $V$ itself has negative polarity.

The trigger gap in hexane broke down at $3 \cdot 1 \mathrm{kV}$.

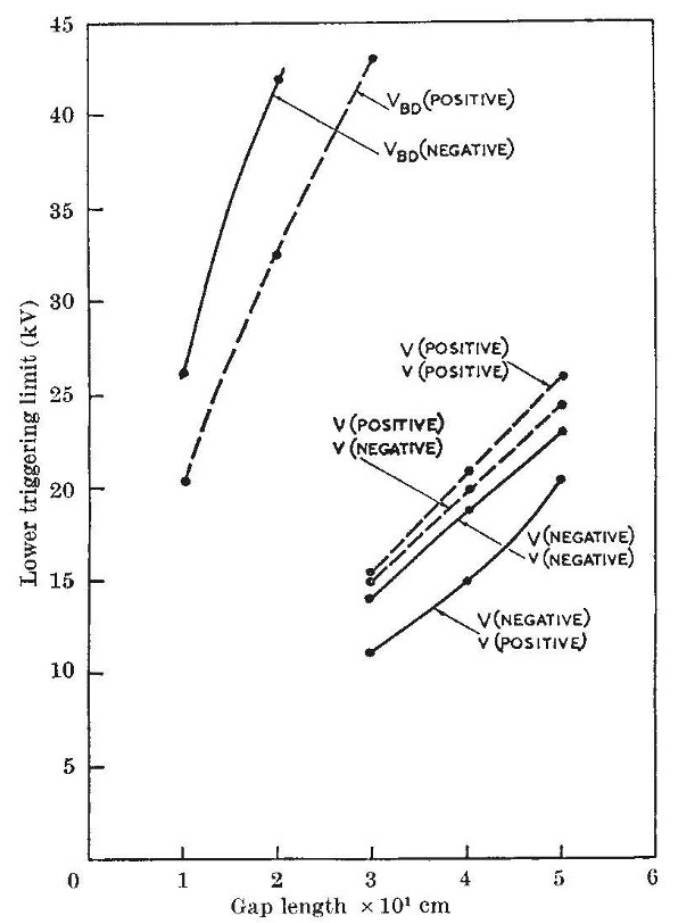

Fig. 1. Lower triggering limit $V_{\min }$. Characteristics for all polarity combinations $(v=5 \mathrm{kV}$ throughout). 